\title{
Study on Acoustic Characteristics of a Flexible Plate Strongly Coupled with Rectangular Cavity
}

\author{
Jinlong Liao $(\mathbb{D}$, Haichao Zhu $(\mathbb{D}$, Jiuxiao Hou, and Suwei Yuan \\ National Key Laboratory on Ship Vibration \& Noise, Institute of Noise \& Vibration, Naval University of Engineering, \\ Wuhan 430033, China \\ Correspondence should be addressed to Haichao Zhu; haiczhu@163.com
}

Received 23 February 2021; Accepted 21 May 2021; Published 31 May 2021

Academic Editor: Davood Younesian

Copyright (c) 2021 Jinlong Liao et al. This is an open access article distributed under the Creative Commons Attribution License, which permits unrestricted use, distribution, and reproduction in any medium, provided the original work is properly cited.

This paper presents a method to predict the acoustic characteristics and steady-state responses of a flexible plate strongly coupled with rectangular cavity based on energy principle theory and Legendre polynomial series. First, the displacement of the plate and the sound pressure in the cavity are constructed in the form of two-dimensional and three-dimensional Legendre polynomial series, respectively. The unknown expansion coefficients are obtained using the Rayleigh-Ritz technique based on the energy expressions for the strongly coupled plate-cavity system. The accuracy, convergence, and efficiency of the present method are verified by comparing with the results available in the FEM and literature. Finally, the effects of the structural boundary conditions, cavity depth, and structural length-width ratio on the coupling natural frequency and the steady-state responses under three excitation conditions are analyzed.

\section{Introduction}

The structure-acoustic interaction system consisting of a flexible vibrating plate and a closed acoustic cavity is widely used in daily life and practical engineering, for instance, bedrooms, aircraft, ship cabins, and sonar platform cavity. In recent years, with the growing requirements for acoustical performance of products as well as comfortable working and living conditions, a clear understanding of acoustic characteristics of structure-acoustic interaction system is the key to acoustical product design and noise control. However, most research is carried out with the assumption of weak coupling between structure and acoustics. With the development of engineering field, the strong coupling situation where, for example, the contained acoustic medium has large mass density, the plate is thin, and the cavity is shallow is becoming more and more common. Therefore, it is of great value to deeply study the acoustic characteristics of the strongly coupled structure-acoustic system.

In practice, a flexible vibrating plate backed by a closed rectangular cavity is a classical representation of numerous engineering backgrounds and has been extensively studied for a long time. In 1963, Lyon [1] and Dowell and Voss [2] firstly studied the acoustic characteristic of a flexible plate coupled with a rectangular cavity and theoretically analyzed the attenuation of internal noise in the cavity. Dowell gave the theoretical solution of structural displacement and sound pressure in form of Fourier series. On this basis, Pretlove $[3,4]$ proposed to calculate the vibration response of the structure by using the structural modes in vacuum and analyzed the effect of the cavity depth on the vibration and acoustical response. In order to express the effect of acoustic cavity on structural vibration, Fahy [5] proposed the concept of modal coupling, which uses modal coupling coefficients to characterize the interaction between structure and cavity. By combining numerical and analytical methods, Qaisi [6] obtained the mass and stiffness matrices of the coupled system and analyzed the effect of cavity depth and boundary conditions on the natural frequencies of the coupled system. Pan and Bies [7] gave a comprehensive literature review of structure-acoustic interaction system and studied the coupling mechanism. Kim and Brennan [8] extended the classical modal coupling theory of structure-acoustic interaction system. A compact matrix form of a plate-cavity 
coupled system was established based on the impedance and mobility method, which can be easily solved in numerical computer software. Using the variation of modal coupling theory, Geng et al. [9] analyzed the response of irregular structure-acoustic interaction system. Luo et al. [10] derived the compact matrix formulation by using Green's function theorem and proposed a numerical simulation method to calculate the response of sound pressure and velocity. Zhang et al. [11] studied vibration characteristics of the laminated plate-cavity coupling system using an improved Fourier method (IFM). Furthermore, they [12] analyzed vibroacoustic property of a laminated rotary plate coupled with an impedance cavity under complex boundary conditions by combining the simplified plate theory (SPT) and the improved Fourier-Ritz method. Lee [13] proposed to examine the effect of cavity length on the natural frequency of a nonlinear panel coupled with extended cavity by using the multilevel residue harmonic balance method.

In addition to the research on weakly coupled panelcavity system, the study of strongly coupled panel-cavity system has attracted the interest of many scholars. Tournour and Atalla [14] analyzed the effect of the truncation value on the response of the strongly coupled plate-cavity system and pointed out that the vibration and sound response of the system could not be accurately predicted by increasing the truncation order in the strongly coupled case and proposed a pseudostatic correction to improve its convergence. David and Menelle [15] proposed a decoupled modal projection method for analyzing the characteristic frequency of the strongly coupled plate-cavity system. In order to investigate the acoustic characteristic of an elastically restrained plate coupled with an enclosure, Du et al. [16-19] proposed an improved Fourier series by introducing auxiliary function to ensure the continuity of vibration velocity on the platecavity interface. Shi et al. [20] used coordinate transformation to map irregular cavities to the unit square domain and studied its acoustic properties based on the three-dimensional improved Fourier series. On the basis, Chen et al. $[21,22]$ modeled the coupling system by replacing the improved Fourier series with Chebyshev polynomial series (CPSM), which was more concise and required less computation. Kim et al. [23] presented a new formulation of the coupled reduced-order modeling technique for fluidstructure interaction problems.

In the view of the existing literatures, different from the research on the weakly coupled panel-cavity system, the research on the strongly coupled plate-cavity system focuses on the system characteristics and less on the steadystate response. Meanwhile, the computational cost of IFM and CPSM is high $[20,21]$. Inspired by these limitations, based on the principle of energy, the acoustical characteristics of the strongly coupled plate-cavity system with arbitrary structure boundary constraints is analyzed by using Legendre polynomial series in this paper. A general expression of structural boundary conditions is established. The structural displacement functions of the plate and sound pressure functions of the cavity are expanded by Legendre polynomial series, respectively. Based on the energy principle, the Lagrange functions of structure and acoustics in the strongly coupled plate-cavity system are developed, respectively. The acoustic characteristics of the system are obtained by the Rayleigh-Ritz technique. Compared with FEM and literatures, the accuracy, convergence, and efficiency of the present method are verified. Finally, the effects of the structural boundary conditions, cavity depth, and structural length-width ratio on the natural frequency are analyzed. On this basis, the steadystate response of the system under force source excitation, internal acoustic source excitation, and a combination of both is studied.

\section{Theoretical Formulations}

2.1. Model Description. As shown in Figure 1, a plate-cavity coupled system consisting of a plate with arbitrary boundary conditions and an acoustic cavity is established to analyze the acoustic characteristics. The rectangular cavity has a length, width, and height of $L_{x}, L_{y}$, and $L_{z}$, respectively, and is filled with a heavy medium (e.g., water) with a mass density of $\rho_{0}$ and a speed of sound propagation of $c_{0}$. The plate has the same length and width as the cavity and is coupled to it at the top surface of the cavity. In addition, the thickness of the plate is denoted as $h$, and the mass density is $\rho_{h}$. A point force source excitation $F_{p}$ on the plate $\left(x_{p}, y_{p}\right)$ and a point sound source excitation $Q_{c}$ at the cavity $\left(x_{c}, y_{c}, z_{c}\right)$ are introduced to analyze the steady-state response of the coupling system.

The strongly coupled plate-cavity system can be divided into the acoustic cavity system and flexible plate system. For the acoustic cavity system, the internal sound pressure satisfies the Helmholtz equation and boundary conditions:

$$
\begin{aligned}
& \nabla^{2} p(x, y, z, t)+\frac{\omega^{2}}{c_{0}^{2}} p(x, y, z, t)=0 \\
& \frac{\partial p(x, y, z, t)}{\partial n}= \begin{cases}\rho_{0} \omega^{2} w(x, y, t), & \text { on the coupling surface, } \\
0, & \text { on the rigid wall, }\end{cases}
\end{aligned}
$$

where $\nabla$ is the Laplace operator, $\omega=2 \pi f$ is the excitation frequency, $w(x, y, t)$ is the structural displacement of the flexible plate, and $n$ is the external normal direction of the flexible plate.

For the flexible plate system, the effect of sound pressure at the interface should be considered as well as the point force source excitation. Based on the theory of structural dynamics, the differential equation for the elastic structural plate takes the following form:

$$
D \nabla^{4} w(x, y, t)+m \frac{\partial^{2} w(x, y, t)}{\partial t^{2}}=p\left(x, y, L_{z}, t\right)-F_{p},
$$

where $D$ and $m$ are the structural bending stiffness and density of plate, respectively. Each boundary constraint of the plate can be described by the linear spring stiffness coefficient $k$ and rotational spring stiffness coefficient $K$; take $x=0$ as an example [16]: 


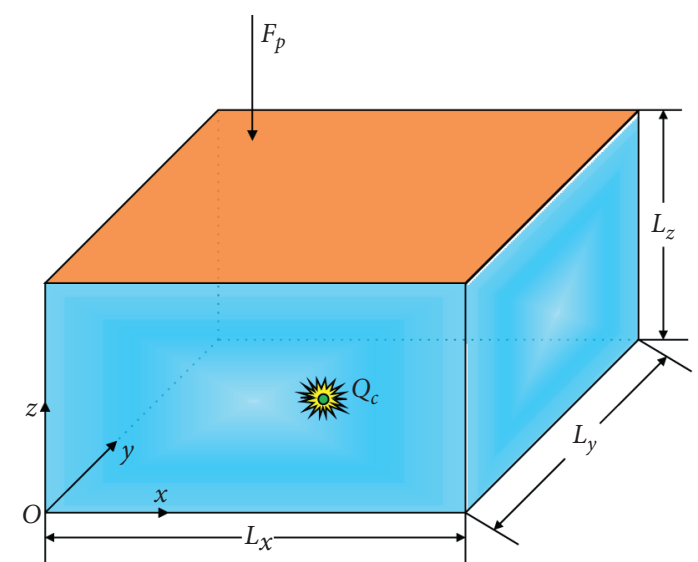

Figure 1: Schematic of the considered flexible plate strongly coupled with a rectangular cavity.

$$
\begin{aligned}
k_{x=0} w(x, y, t) & =-D\left(\frac{\partial^{3} w(x, y, t)}{\partial x^{3}}+(2-\eta) \frac{\partial^{3} w(x, y, t)}{\partial x \partial y^{2}}\right), \\
K_{x=0} \frac{\partial w(x, y, t)}{\partial x} & =D\left(\frac{\partial^{2} w(x, y, t)}{\partial x^{2}}+\eta \frac{\partial^{2} w(x, y, t)}{\partial y^{2}}\right)
\end{aligned}
$$

where $\eta$ is Poisson's ratio and $k_{x=0}$ and $K_{x=0}$ are the linear spring stiffness coefficient and rotational spring stiffness coefficient, respectively. The variation of the elastic boundary constraints can be realized by setting the stiffness coefficient to zero or infinity [16]. For example, if the linear spring stiffness coefficient is set to infinity (which is represented by $10^{11}$ in following numerical calculations) and the rotational spring stiffness coefficient is set to zero, the simply supported boundary condition can be obtained. If the linear spring stiffness coefficient and the rotational spring stiffness coefficient are both set to infinity, the clamped boundary conditions can be obtained.

2.2. Series Representation of Sound Pressure and Structural Displacement Functions. The velocity continuity will not be satisfied at the coupling interface with the strong coupling between plate and cavity if the rigidly walled cavity mode is used to represent the sound pressure as well as the vacuum structure mode is used to represent the displacement. In order to overcome the problem, the structural displacement and sound pressure are expanded by Legendre polynomial series; in addition, coordinate transformation is required since the Legendre polynomials define the interval as $[-1,1]$ :

$$
\begin{aligned}
& \alpha=\frac{2 x}{L_{x}}-1, \\
& \beta=\frac{2 y}{L_{y}}-1, \\
& \gamma=\frac{2 z}{L_{z}}-1 .
\end{aligned}
$$

The sound pressure and structural displacement functions can then be expressed as

$$
\begin{aligned}
p(\alpha, \beta, \gamma, t) & =e^{-j w t}\left(\sum_{m_{\alpha}=0}^{\infty} \sum_{m_{\beta}=0}^{\infty} \sum_{m_{\gamma}=0}^{\infty} A_{m_{\alpha} m_{\beta} m_{\gamma}} P_{m_{\alpha}}(\alpha) P_{m_{\beta}}(\beta) P_{m_{\gamma}}(\gamma)\right) \\
w(\alpha, \beta, t) & =e^{-j w t}\left(\sum_{n_{\alpha}=0}^{\infty} \sum_{n_{\beta}=0}^{\infty} B_{n_{\alpha} n_{\beta}} P_{n_{\alpha}}(\alpha) P_{n_{\beta}}(\beta)\right)
\end{aligned}
$$

$$
\int_{-1}^{1} P_{m_{\alpha}}(\alpha) P_{m_{\alpha}^{\prime}}(\alpha)=\frac{2}{2 m_{\alpha}+1} \delta_{m_{\alpha} m_{\alpha}^{\prime}}
$$

where $A_{m_{\alpha} m_{\beta} m_{\gamma}}$ is the $\left(m_{\alpha}, m_{\beta}, m_{\gamma}\right)$ th term of the expansion coefficient vectors of sound pressure $\mathbf{A}$ and $B_{n_{\alpha} n_{\beta}}$ is the $\left(n_{\alpha}, n_{\beta}\right)$ th term of the expansion coefficient vectors of structural displacement $\mathbf{B}$. The time item $e^{-j w t}$ will be omitted below for simplicity; $P_{m_{\alpha}}(\alpha)$ is Legendre polynomial [24] with the form as

$$
P_{m_{\alpha}}(\alpha)=\frac{1}{2^{m_{\alpha}} m_{\alpha} !} \frac{d^{m_{\alpha}}}{d \alpha^{m_{\alpha}}}\left[\left(\alpha^{2}-1\right)^{m_{\alpha}}\right] .
$$

It should be noted that although the infinite polynomial expansion terms are included in equations (5) and (6), only the first finite polynomials will be used in numerical calculations. In addition, Legendre polynomials are not only complete and recursive as other polynomials, but also their L2 inner product satisfies orthogonality: where $\delta_{m_{\alpha} m_{\alpha}^{\prime}}$ is the Kronecker function, only when $m_{\alpha}=m_{\alpha}^{\prime}$, $\delta_{m_{\alpha} m_{\alpha}^{\prime}}$ is 1 , otherwise, $\delta_{m_{\alpha} m_{\alpha}^{\prime}}$ is 0 .

2.3. Energy Expression of Acoustic Cavity System and Flexible Plate System. Since the strongly coupled plate-cavity system can be divided into the acoustic cavity system and the flexible plate system, based on the energy principle, the Lagrange functions $[18,19]$ of the acoustic cavity system and the flexible plate system can be obtained, respectively. For the acoustic cavity system:

$$
L_{C}=U_{C}-T_{C}-W_{Q}-W_{P 2 C},
$$

where $U_{C}$ is the total acoustic potential energy of the acoustic cavity, $T_{C}$ is the total kinetic energy of the acoustic 
cavity, $W_{Q}$ is the work done by the point sound source, and $W_{P 2 C}$ represents the work done by structural vibration to the medium in the cavity [16]. Their specific expressions are

$$
\begin{aligned}
U_{C} & =\frac{L_{x} L_{y} L_{z}}{16 \rho c^{2}} \int_{-1}^{1} \int_{-1}^{1} \int_{-1}^{1} p^{2}(\alpha, \beta, \gamma) \mathrm{d} \alpha \mathrm{d} \beta \mathrm{d} \gamma \\
T_{C} & =\frac{L_{x} L_{y} L_{z}}{4 \rho \omega^{2}} \int_{-1}^{1} \int_{-1}^{1} \int_{-1}^{1}\left[\left(\frac{\partial p(\alpha, \beta, \gamma)}{L_{x} \partial \alpha}\right)^{2}+\left(\frac{\partial p(\alpha, \beta, \gamma)}{L_{y} \partial \beta}\right)^{2}+\left(\frac{\partial p(\alpha, \beta, \gamma)}{L_{z} \partial \gamma}\right)^{2}\right] \mathrm{d} \alpha \mathrm{d} \beta \mathrm{d} \gamma \\
W_{Q} & =-\frac{1}{j \omega} Q_{c} p\left(\alpha_{c}, \beta_{c}, \gamma_{c}\right), \\
W_{P 2 C} & =\frac{L_{x} L_{y}}{4} \int_{-1}^{1} \int_{-1}^{1} w(\alpha, \beta) p(\alpha, \beta, 1) \mathrm{d} \alpha \mathrm{d} \beta .
\end{aligned}
$$

For the flexible plate system, the Lagrange function expression $[18,19]$ is shown as below:

$$
L_{P}=U_{P}-T_{P}-W_{P}-W_{C 2 P},
$$

where $U_{P}$ is the total acoustic potential energy of the flexible plate, $T_{P}$ is the total kinetic energy of the flexible plate, $W_{P}$ is the work done by the point force source, and $W_{C 2 P}$ represents the work done by sound pressure to the flexible plate [20]. Their specific expressions are

$$
\begin{aligned}
U_{P}= & \frac{D}{2} \int_{-1}^{1} \int_{-1}^{1} \frac{4 L_{y}}{L_{x}^{3}}\left(\frac{\partial^{2} w(\alpha, \beta)}{\partial \alpha^{2}}\right)^{2}+\frac{4 L_{x}}{L_{y}^{3}}\left(\frac{\partial^{2} w(\alpha, \beta)}{\partial \beta^{2}}\right)^{2}+\frac{8 \eta}{L_{x} L_{y}}\left(\frac{\partial^{2} w(\alpha, \beta)}{\partial \alpha^{2}} \frac{\partial^{2} w(\alpha, \beta)}{\partial \beta^{2}}\right)+\frac{8(1-\eta)}{L_{x} L_{y}}\left(\frac{\partial^{2} w(\alpha, \beta)}{\partial \alpha^{2} \partial \beta^{2}}\right)^{2} \mathrm{~d} \alpha \mathrm{d} \beta \\
& +\frac{L_{x}}{4} \int_{-1}^{1}\left[k_{y 0} w^{2}(\alpha, \beta)+\frac{4}{L_{y}^{2}} K_{y 0}\left(\frac{\partial w(\alpha, \beta)}{\partial \beta}\right)^{2}\right]_{\beta=-1} \mathrm{~d} \alpha+\frac{L_{x}}{4} \int_{-1}^{1}\left[k_{y L_{y}} w^{2}(\alpha, \beta)+\frac{4}{L_{y}^{2}} K_{y L_{y}}\left(\frac{\partial w(\alpha, \beta)}{\partial \beta}\right)^{2}\right]_{\beta=1} \mathrm{~d} \alpha \\
& +\frac{L_{y}}{4} \int_{-1}^{1}\left[k_{x 0} w^{2}(\alpha, \beta)+\frac{4}{L_{x}^{2}} K_{x 0}\left(\frac{\partial w(\alpha, \beta)}{\partial \alpha}\right)^{2}\right]_{\alpha=-1} \mathrm{~d} \beta+\frac{L_{y}}{4} \int_{-1}^{1}\left[k_{x L_{x}} w^{2}(\alpha, \beta)+\frac{4}{L_{x}^{2}} K_{x L_{x}}\left(\frac{\partial w(\alpha, \beta)}{\partial \alpha}\right)^{2}\right] \mathrm{d} \beta, \\
T_{P}= & \frac{L_{x} L_{y}}{8} \rho h \omega^{2} \int_{-1}^{1} \int_{-1}^{1} w(\alpha, \beta) \mathrm{d} \alpha \mathrm{d} \beta \\
W_{P}= & w\left(\alpha_{p}, \beta_{p}\right) F_{P}, \\
W_{C 2 P}= & -W_{P 2 C} .
\end{aligned}
$$

2.4. Solution Method. Substitute equation (5) and equations (10) (13) into equation (9). Substitute equation (6) and equations (15) (18) into equation (14). According to the Rayleigh-Ritz method $[25,26]$, set the partial derivative of the Lagrange functions $L_{C}$ with respect to expansion coefficient vectors of sound pressure $\mathbf{A}$ and the Lagrange functions $L_{P}$ with respect to expansion coefficient vectors of structural displacement B as zero, respectively, 


$$
\begin{aligned}
& \frac{\partial L_{C}}{\partial \mathbf{A}}=\mathbf{0} \\
& \frac{\partial L_{P}}{\partial \mathbf{B}}=\mathbf{0} .
\end{aligned}
$$

The linear algebraic equations of the expansion coefficient vectors $\mathbf{A}$ and $\mathbf{B}$ can be obtained:

$$
\begin{gathered}
\left(\mathbf{K}_{P}-\omega^{2} \mathbf{M}_{P}\right) \mathbf{B}+\mathbf{K}_{C 2 P} \mathbf{A}=\mathbf{F}, \\
\left(\mathbf{K}_{C}-\omega^{2} \mathbf{M}_{C}\right) \mathbf{A}+\omega^{2} \mathbf{K}_{P 2 C} \mathbf{B}=\mathbf{Q} .
\end{gathered}
$$

The overall form can be developed by combining equations (20) and (21):

$$
\left\{\left[\begin{array}{cc}
\mathbf{K}_{P} & \mathbf{K}_{C 2 P} \\
\mathbf{0} & \mathbf{K}_{C}
\end{array}\right]-\omega^{2}\left[\begin{array}{cc}
\mathbf{M}_{P} & \mathbf{0} \\
-\mathbf{K}_{P 2 C} & \mathbf{M}_{C}
\end{array}\right]\right\}\left[\begin{array}{l}
\mathbf{B} \\
\mathbf{A}
\end{array}\right]=\left[\begin{array}{l}
\mathbf{F} \\
\mathbf{Q}
\end{array}\right],
$$

where $\mathbf{K}_{P}$ and $\mathbf{K}_{C}$ are the stiffness matrices of the acoustic cavity system and the flexible plate system, $\mathbf{M}_{P}$ and $\mathbf{M}_{C}$ are the mass matrices of the acoustic cavity system and the flexible plate system, $\mathbf{K}_{C 2 P}$ represents the coupling effect matrix of the acoustic cavity system to the flexible plate system, and $\mathbf{K}_{P 2 C}$ represents the coupling effect matrix of the flexible plate system to the acoustic cavity system.

According to the principle of action and reaction, we can get the equality equation of $\mathbf{K}_{P 2 C}=\mathbf{K}_{C 2 P}^{T}$. $\mathbf{F}$ is is the force vector which acted on the flexible plate and $\mathbf{Q}$ is the sound source vector of the internal point sound source. The acoustic characteristics of the strongly coupled plate-cavity system can be obtained by setting $\mathbf{F}$ and $\mathbf{Q}$ to zero. On the contrary, when considering the external force vectors $\mathbf{F}$ and $\mathbf{Q}$, the steady-state responses of the coupling system can be developed. Since the L2 inner product of Legendre polynomial satisfies the orthogonality, the high-multiple integral term in each matrix element can be simplified to lowmultiple integral or direct product term. According to equation (8), the matrix element can be simplified into the following formulae:

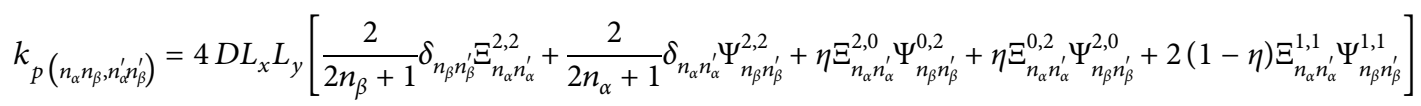

$$
\begin{aligned}
& +\frac{L_{x}}{2 n_{\alpha}+1} \delta_{n_{\alpha} n_{\alpha}^{\prime}}\left[k_{y 0} P_{n_{\beta}}(-1) P_{n_{\beta}^{\prime}}(-1)+\frac{4}{L_{y}^{2}} K_{y 0} P_{n_{\beta}}^{\prime}(-1) P_{n_{\beta}^{\prime}}^{\prime}(-1)+k_{y L y} P_{n_{\beta}}(1) P_{n_{\beta}^{\prime}}(1)+\frac{4}{L_{y}^{2}} K_{y L y} P_{n_{\beta}^{\prime}}(1) P_{n_{\beta}^{\prime}}^{\prime}(1)\right] \\
& +\frac{L_{y}}{2 q+1} \delta_{n_{\beta} n_{\beta}^{\prime}}\left[k_{x 0} P_{n_{\alpha}}(-1) P_{n_{\alpha}^{\prime}}(-1)+\frac{4}{L_{x}^{2}} K_{x 0} P_{n_{\alpha}}^{\prime}(-1) P_{n_{\alpha}^{\prime}}^{\prime}(-1)+k_{x L x} P_{n_{\alpha}}(1) P_{n_{\alpha}^{\prime}}(1)+\frac{4}{L_{x}^{2}} K_{x L x} P_{n_{\alpha}}^{\prime}(1) P_{n_{\alpha}^{\prime}}^{\prime}(1)\right] \text {, } \\
& k_{c\left(m_{\alpha} m_{\beta} m_{\gamma}, m_{\alpha}^{\prime} m_{\beta}^{\prime} m_{\gamma}^{\prime}\right)}=\frac{L_{x} L_{y} L_{z}}{\rho_{0}}\left[\frac{2}{L_{x}^{2}\left(2 m_{\beta}+1\right)\left(2 m_{\gamma}+1\right)} \delta_{m_{\beta} m_{\beta}^{\prime}} \delta_{m_{\gamma} m_{\gamma}^{\prime} \Xi_{m_{\alpha} m_{\alpha}^{\prime}}^{1,1}}\right. \\
& +\frac{2}{L_{y}^{2}\left(2 m_{\alpha}+1\right)\left(2 m_{\gamma}+1\right)} \delta_{m_{\alpha} m_{\alpha}^{\prime}} \delta_{m_{\gamma} m_{\gamma}^{\prime} \Psi_{m_{\beta} m_{\beta}^{\prime}}^{1,1}}
\end{aligned}
$$

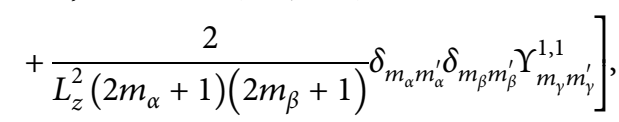

$$
\begin{aligned}
& m_{p\left(n_{\alpha} n_{\beta}, n_{\alpha}^{\prime} n_{\beta}^{\prime}\right)}=\frac{L_{x} L_{y} \rho_{h} h}{\left(2 n_{\alpha}+1\right)\left(2 n_{\beta}+1\right)} \delta_{n_{\alpha} n_{\alpha}^{\prime}} \delta_{n_{\beta} n_{\beta}^{\prime}} \\
& m_{c\left(m_{\alpha} m_{\beta} m_{\gamma}, m_{\alpha}^{\prime} m_{\beta}^{\prime} m_{\gamma}^{\prime}\right)}=\frac{L_{x} L_{y} L_{z}}{\rho_{0} c_{0}^{2}\left(2 m_{\alpha}+1\right)\left(2 m_{\beta}+1\right)\left(2 m_{\gamma}+1\right)} \delta_{m_{\alpha} m_{\alpha}^{\prime}} \delta_{m_{\beta} m_{\beta}^{\prime}} \delta_{m_{\gamma} m_{\gamma}^{\prime}}, \\
& k_{p 2 c\left(m_{\alpha} m_{\beta} m_{\gamma}, n_{\alpha} n_{\beta}\right)}=\frac{L_{x} L_{y}}{\left(2 n_{\alpha}+1\right)\left(2 n_{\beta}+1\right)} \delta_{n_{\alpha} m_{\alpha}} \delta_{n_{\beta} m_{\beta}} P_{m_{\gamma}}(1) \\
& f_{n_{\alpha} n_{\beta}}=F_{P} P_{n_{\alpha}}\left(\alpha_{p}\right) P_{n_{\beta}}\left(\beta_{p}\right), \\
& q_{m_{\alpha} m_{\beta} m_{\gamma}}=-j \omega Q_{c} P_{m_{\alpha}}\left(\alpha_{c}\right) P_{m_{\beta}}\left(\beta_{c}\right) P_{m_{\gamma}}\left(\gamma_{c}\right) \text {, }
\end{aligned}
$$


where

$$
\begin{aligned}
& \Xi_{a b}^{v, \bar{v}}=\int_{-1}^{1} \frac{d^{v} P_{a}^{v}(\alpha)}{L_{x}^{v} d \alpha^{v}} \frac{d^{\bar{v}} P_{a}^{\bar{v}}(\alpha)}{L_{x}^{\bar{v}} \mathrm{~d} \alpha^{\bar{v}}}\left(a=n_{\alpha}, m_{\alpha} ; b=n_{\alpha}^{\prime}, m_{\alpha}^{\prime}\right) \\
& \Psi_{a b}^{v, \bar{v}}=\int_{-1}^{1} \frac{d^{v} P_{a}^{v}(\beta)}{L_{y}^{v} d \beta^{v}} \frac{d^{\bar{v}} P_{a}^{\bar{v}}(\beta)}{L_{y}^{\bar{v}} d \beta^{\bar{v}}}\left(a=n_{\beta}, m_{\beta} ; b=n_{\beta}^{\prime}, m_{\beta}^{\prime}\right) \\
& \Upsilon_{a, b}^{v, \bar{v}}=\int_{-1}^{1} \frac{d^{v} P_{a}^{v}(\gamma)}{L_{z}^{v} d \gamma^{v}} \frac{d^{\bar{v}} P_{a}^{\bar{v}}(\gamma)}{L_{z}^{\bar{v}} d \gamma^{\bar{v}}}\left(a=m_{\gamma} ; b=m_{\gamma}^{\prime}\right)
\end{aligned}
$$

\section{Method Validation}

The accuracy and convergence of the method are verified by comparing with the results available in the FEM and literature. The FEM model is established in COMSOL, and the sound-shell coupling module is used to model the strongly coupled plate-cavity system. To obtain reliable results, the maximum mesh size is set to $1 / 6$ of the minimum wavelength of the analysis band. A water-filled cavity bounded by a simply supported plate is considered as an analysis model which has been studied in Ref [21]. The specific parameters of the plate-cavity system are shown in Table 1 .

3.1. Accuracy and Convergence Analysis. Table 2 shows the first eight natural frequencies of the strongly coupled platecavity system. Different truncation values are selected for sound pressure expression (represented by $m_{\alpha}, m_{\beta}, m_{\gamma}$ ) and structural displacement expression (represented by $n_{\alpha}, n_{\beta}$ ) to analyze the accuracy and convergence of the present method. It can be seen from Table 2 that with the increase of the truncation values of the sound pressure expression and structural displacement expression, the results of present method converge rapidly and are in good agreement with the results available in the FEM and literature.

Figure 2 shows the first five modes of displacement distribution of the plate calculated by FEM and the present method with the truncation values values $m_{\alpha}=m_{\beta}=m_{\gamma}=10, n_{\alpha}=n_{\beta}=10$. In addition to accurately expressing the displacement distribution of the structure, we can also find that the strong coupling between plate and cavity caused by the incompressibility of the heavy fluid medium changes the asymmetric mode displacement distribution of the plate. Different from the structural displacement distribution in vacuum [27], where the first piston-like mode in vacuum is replaced by the symmetric mode, the fourth and fifth modes (the fifth and sixth modes in vacuum) have obvious changes, and the second and third modes (the third and fourth modes in vacuum) basically have no effect. The incompressibility of the heavy fluid medium leads to significant changes in the asymmetrically distributed modes, while the symmetrically distributed modes counteract the effects of coupling.
TABLE 1: The model parameters.

\begin{tabular}{lcc}
\hline & Length $(\mathrm{m})$ & 0.29 \\
Cavity & Width $(\mathrm{m})$ & 0.35 \\
& Depth $(\mathrm{m})$ & 0.14 \\
& Water density $\left(\mathrm{kg} \cdot \mathrm{m}^{-3}\right)$ & 1000 \\
& Sound velocity $\left(\mathrm{m} \cdot \mathrm{s}^{-1}\right)$ & 1500 \\
\hline \multirow{4}{*}{ Plate } & Length $(\mathrm{m})$ & 0.29 \\
& Width $(\mathrm{m})$ & 0.35 \\
& Thickness $(\mathrm{m})$ & 0.0015 \\
& Poisson's ratio & 0.3 \\
& Young's modulus $(\mathrm{Pa})$ & $7.2 \times 10^{10}$ \\
& Mass density $\left(\mathrm{kg} \cdot \mathrm{m}^{-3}\right)$ & 2700 \\
\hline
\end{tabular}

3.2. Computational Efficiency Analysis. In contrast to Ref [21], which proposed to expand the structural displacement function and the sound pressure function with Chebyshev polynomial series, this paper uses Legendre polynomial series to describe the sound pressure and displacement. Since the L2 inner product of Legendre polynomial satisfies the orthogonality, it will simplify the calculation. For code implementations of the two methods on computers with the same computing power, the main influence on the computational efficiency is the dimensionality of one-dimension integration terms. The appendix gives expressions for each element of the stiffness matrix, mass matrix, and coupling interaction matrix from Ref [21]. For the same analytical model with the same truncation values, the total dimensionality of the onedimension integral terms for present method and Ref [21] can be obtained according to equations (23)-(32) and the Appendix:

$$
\begin{aligned}
& \operatorname{Dim}_{\text {pre }}=n_{\alpha}^{2}+n_{\beta}^{2}+3 n_{\alpha}^{2} n_{\beta}^{2}+m_{\alpha}^{2}+m_{\beta}^{2}+m_{\gamma}^{2}, \\
& \operatorname{Dim}_{\text {ref }}=6 n_{\alpha}^{2} n_{\beta}^{2}+4 m_{\alpha}^{2} m_{\beta}^{2} m_{\gamma}^{2}+n_{\alpha}^{2}+n_{\beta}^{2}+n_{\alpha} m_{\alpha} n_{\beta} m_{\beta} .
\end{aligned}
$$

It can be seen from equations (33) and (34) that the total dimensionality of the one-dimension integral term is greatly reduced, making the code easier to handle and improving computational efficiency when using Legendre polynomial series to describe sound pressure and structural displacement.

\section{Numerical Results and Discussion}

4.1. Natural Characteristics Analysis. In this section, the effects of the structural boundary conditions, cavity depth, and structural length-width ratio on the natural frequency of simply supported strongly coupled plate-cavity system are studied. The model parameters are shown in Table 1, which are used in subsequent calculation. Considering the correctness of the results and the efficiency of the calculation, the truncation values can be set to $m_{\alpha}=m_{\beta}=m_{\gamma}=10$ and $n_{\alpha}=n_{\beta}=10$ in the following numerical calculations.

4.1.1. Effects of Structural Boundary Conditions. In this section, the natural frequencies under different boundary conditions are analyzed by changing the spring stiffness 
TABLE 2: The first eight natural frequencies of the strongly coupled plate-cavity system.

\begin{tabular}{|c|c|c|c|c|c|c|c|c|c|}
\hline \multirow{2}{*}{$m_{\alpha} \times m_{\beta} \times m_{\gamma}$} & \multirow{2}{*}{$n_{\alpha} \times n_{\beta}$} & \multicolumn{8}{|c|}{ Mode number } \\
\hline & & 1 & 2 & 3 & 4 & 5 & 6 & 7 & 8 \\
\hline \multirow{5}{*}{$8 \times 8 \times 8$} & $6 \times 6$ & 33.564 & 46.523 & 78.589 & 112.209 & 163.658 & 171.812 & 207.975 & 269.727 \\
\hline & $8 \times 8$ & 33.548 & 46.580 & 78.575 & 85.232 & 125.997 & 137.665 & 162.189 & 175.863 \\
\hline & $10 \times 10$ & 33.547 & 46.579 & 78.572 & 84.802 & 125.100 & 137.282 & 161.111 & 172.606 \\
\hline & $12 \times 12$ & 33.547 & 46.579 & 78.572 & 84.797 & 125.090 & 137.275 & 161.095 & 172.552 \\
\hline & $14 \times 14$ & 33.547 & 46.579 & 78.572 & 84.797 & 125.090 & 137.275 & 161.094 & 172.552 \\
\hline \multirow{5}{*}{$10 \times 10 \times 10$} & $6 \times 6$ & 33.564 & 46.622 & 78.587 & 112.179 & 163.590 & 171.759 & 207.902 & 269.588 \\
\hline & $8 \times 8$ & 33.548 & 46.580 & 78.574 & 85.211 & 125.963 & 137.635 & 162.141 & 175.742 \\
\hline & $10 \times 10$ & 33.547 & 46.578 & 78.571 & 84.764 & 125.033 & 137.231 & 160.992 & 172.268 \\
\hline & $12 \times 12$ & 33.547 & 46.578 & 78.571 & 84.757 & 125.020 & 137.220 & 160.965 & 172.175 \\
\hline & $14 \times 14$ & 33.547 & 46.578 & 78.571 & 84.757 & 125.019 & 137.220 & 160.965 & 172.174 \\
\hline \multirow{5}{*}{$12 \times 12 \times 12$} & $6 \times 6$ & 33.564 & 46.622 & 78.587 & 112.176 & 163.582 & 171.754 & 207.894 & 269.571 \\
\hline & $8 \times 8$ & 33.547 & 46.580 & 78.573 & 85.211 & 125.962 & 137.634 & 162.140 & 175.741 \\
\hline & $10 \times 10$ & 33.547 & 46.578 & 78.571 & 84.764 & 125.032 & 137.229 & 160.991 & 172.265 \\
\hline & $12 \times 12$ & 33.547 & 46.578 & 78.571 & 84.757 & 125.018 & 137.218 & 160.962 & 172.167 \\
\hline & $14 \times 14$ & 33.547 & 46.578 & 78.570 & 84.757 & 125.018 & 137.218 & 160.962 & 172.166 \\
\hline FEM & & 33.509 & 46.513 & 78.413 & 84.577 & 124.670 & 136.810 & 160.420 & 171.570 \\
\hline $\operatorname{Ref}[21]$ & & 33.574 & 46.596 & 78.608 & 84.102 & 124.680 & 137.112 & 161.082 & 172.334 \\
\hline
\end{tabular}

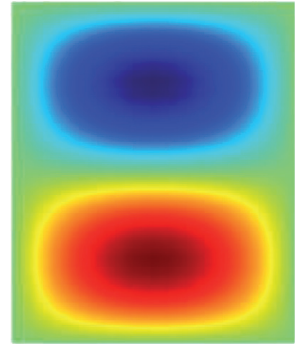

1st mode

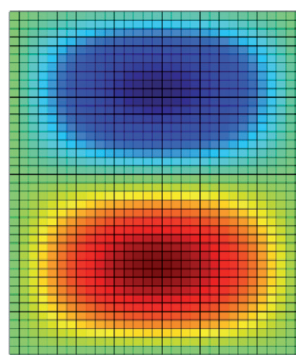

1st mode

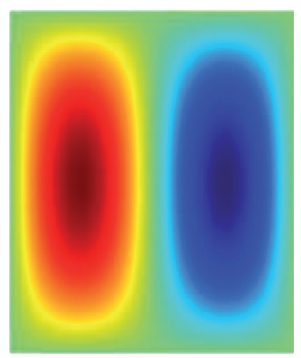

2nd mode

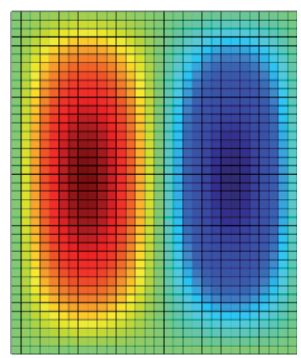

2nd mode

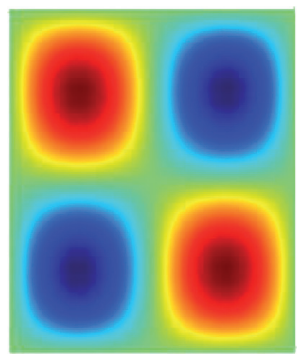

3rd mode

(a)

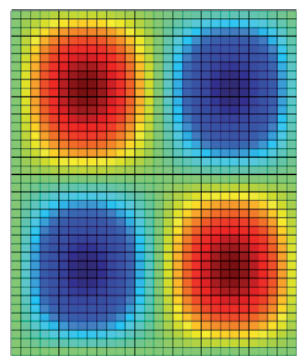

3rd mode

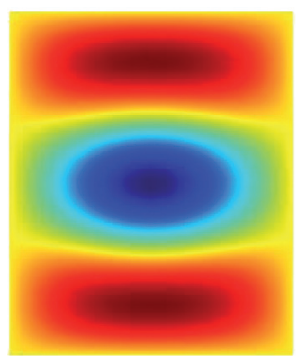

4th mode

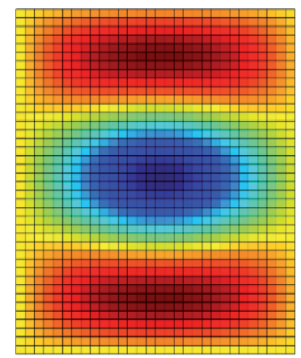

4th mode

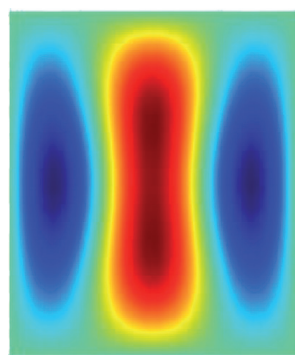

5th mode

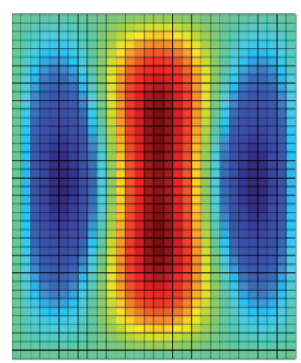

5 th mode

(b)

FIGURE 2: The first five structural modes of the strongly coupled panel-cavity system. (a) FEM. (b) Present method.

coefficient. " $C$ " represents clamped boundary condition and " $S$ " represents simply supported boundary condition. For example, "CSSC" means that $x=0$ is clamped supported, $x=L_{x}$ is simply supported, $y=0$ is simply supported, and $y=L_{y}$ is clamped supported. Table 3 shows the natural frequency under different boundary conditions, and the finite element results are selected as the reference results. For different boundary conditions, the results of the present method and FEM are in good agreement, which on the one hand further demonstrates the accuracy of present method in predicting the acoustic characteristics of strongly coupled systems. On the other hand, the present method is more flexible than traditional methods because it only needs to change the spring stiffness coefficients to achieve different combinations of boundary conditions. In addition, we can also find that the natural frequency of the coupled system under the clamped supported boundary is higher than that of the simply supported boundary. With the increase of the 
TABLE 3: Natural frequencies under different boundary conditions.

\begin{tabular}{lccccccccc}
\hline \multirow{2}{*}{ Boundary conditions } & \multirow{2}{*}{ Results } & \multicolumn{5}{c}{ Mode number } \\
& & 1 & 2 & 3 & 4 & 5 & 6 & 7 \\
\hline \multirow{2}{*}{ SSSS } & Present method & 33.547 & 46.578 & 78.571 & 84.764 & 125.033 & 137.231 & 160.992 & 172.268 \\
& FEM & 33.509 & 46.513 & 78.413 & 84.577 & 124.670 & 136.810 & 160.420 & 171.570 \\
\hline \multirow{2}{*}{ SSSC } & Present method & 40.389 & 48.410 & 85.340 & 96.514 & 127.324 & 150.292 & 168.131 & 191.673 \\
& FEM & 40.309 & 48.330 & 85.109 & 96.178 & 126.930 & 149.650 & 167.440 & 190.370 \\
\hline \multirow{2}{*}{ SSCC } & Present method & 48.625 & 50.755 & 93.262 & 108.600 & 130.969 & 164.836 & 175.427 & 213.522 \\
& FEM & 48.480 & 50.651 & 92.932 & 108.070 & 130.500 & 163.880 & 174.590 & 211.580 \\
\hline \multirow{2}{*}{ SCCC } & Present method & 51.439 & 62.058 & 104.229 & 111.989 & 150.289 & 175.091 & 194.538 & 217.899 \\
& FEM & 51.270 & 61.869 & 103.770 & 111.400 & 149.510 & 173.960 & 193.280 & 215.930 \\
\hline \multirow{2}{*}{ CCCC } & Present method & 55.198 & 75.789 & 115.662 & 117.261 & 172.088 & 187.087 & 210.256 & 228.636 \\
& FEM & 54.993 & 75.459 & 115.010 & 116.620 & 170.820 & 185.720 & 208.430 & 226.560 \\
\hline
\end{tabular}

number of the clamped supported boundary, the natural frequency gradually increases.

4.1.2. Effects of the Cavity Depth. The natural frequencies in various cavity depths in the range of $0.01-1.0 \mathrm{~m}$ are studied. It should be noted that since the cavity depth is variable, the truncation value in the direction of cavity depth is selected as follows: when the cavity depth is less than $0.5 \mathrm{~m}, m_{\gamma}=10$; otherwise, $m_{\gamma}=15$. Figure 3 shows the first eight natural frequency curves in different cavity depths. It can be seen that the frequency variation trend in different order is consistent. When the cavity depth is shallow, the frequency gradually increases with the increase of the cavity depth. As the cavity depth deepens further, the frequency does not change. In addition, it can be found that the frequency variation of the higher order is larger than that of the lower order under the same cavity depth variation.

\subsubsection{Effects of Structural Length-Width Ratio. Keeping} cavity depth and the length of plate constant, Figure 4 shows the variation of the first eight natural frequencies of the plate-cavity system under different length-width ratios $\left(L_{y} / L_{x}\right)$. As the width of the plate changes, the truncation value in this direction should also change as follows: when the length is less than $0.5 \mathrm{~m}, m_{\beta}=n_{\beta}=10$, and when the length is less than $1 \mathrm{~m}$ but is greater than $0.5 \mathrm{~m}$, $m_{\beta}=n_{\beta}=15$; otherwise, $m_{\beta}=n_{\beta}=20$. It can be seen from Figure 4 that the different order natural frequency variation rules of the coupled system under different length-width ratios are relatively consistent. With the increase of lengthwidth ratio, the coupling frequency decreases gradually until it remains constant. In addition, when the lengthwidth ratio is small, the coupling frequency changes more with the length-width ratio. We can slightly adjust the natural frequency of the coupling system by changing the length-width ratio.

4.2. Steady-State Response Analysis. On the basis of the above results, the steady-state responses of the plate-cavity system are studied in this section under three excitation conditions, which are point force source excitation, internal point acoustic source excitation, and a combination of both.

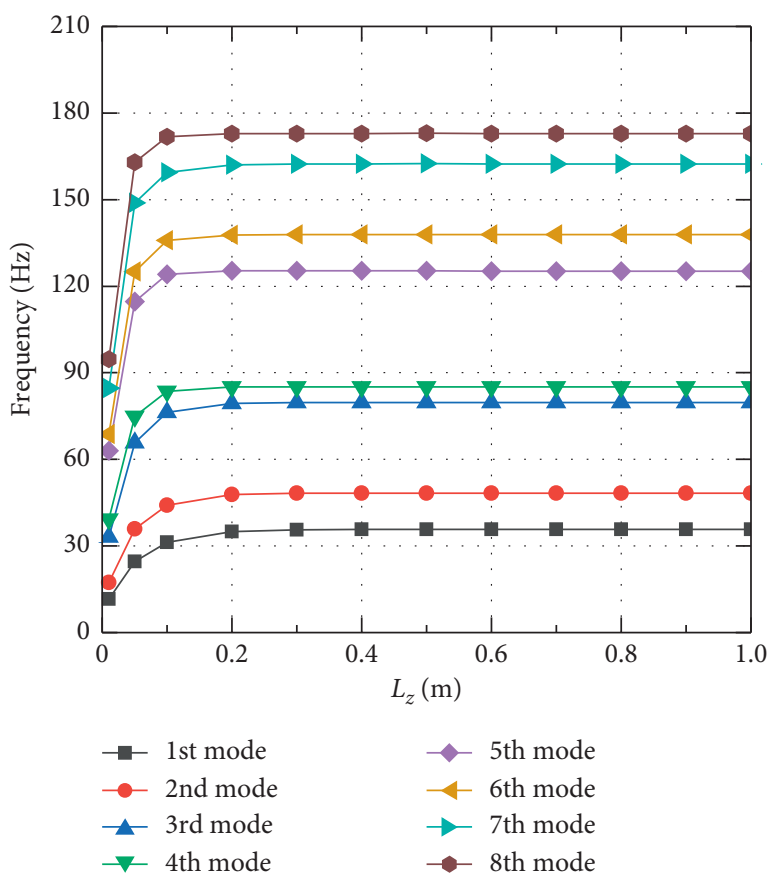

FIGURE 3: Variation of the first eight-order coupling frequency with various cavity depths.

The model parameters are shown in Table 1 . The point force is applied at the thin rectangular plate at $(0.04,0.08,0.14)$ with the magnitude $F=1 \mathrm{~N}$, and the internal point acoustic source is applied at $(0.17,0.20,0.10)$ with the volume velocity $Q_{c}=2 \times 10^{-5} \mathrm{~m}^{3} \cdot \mathrm{s}^{-1}$. Two observation points are set at $(0.10,0.12,0.14)$ and $(0.15,0.30,0.14)$ of the plate to obtain the vibrating velocity response. Similarly, two observation points are set in the cavity at $(0.08,0.11,0.07)$ and $(0.20,0.33$, $0.06)$ to obtain the sound pressure response. In response calculation, structural damping $\xi$ and acoustic medium damping $\zeta$ are both 0.01 , which can be achieved by setting complex Young's modulus as $7.2 \times 10^{10} \times(1-\xi)$ and complex sound velocity as $(1500 /(1-\zeta))$, respectively. Figures 5-7 show the vibrating velocity response and the sound pressure response under three excitation conditions in the frequency range of $0-350 \mathrm{~Hz}$. It can be seen that no matter the point force excitation, internal acoustic 


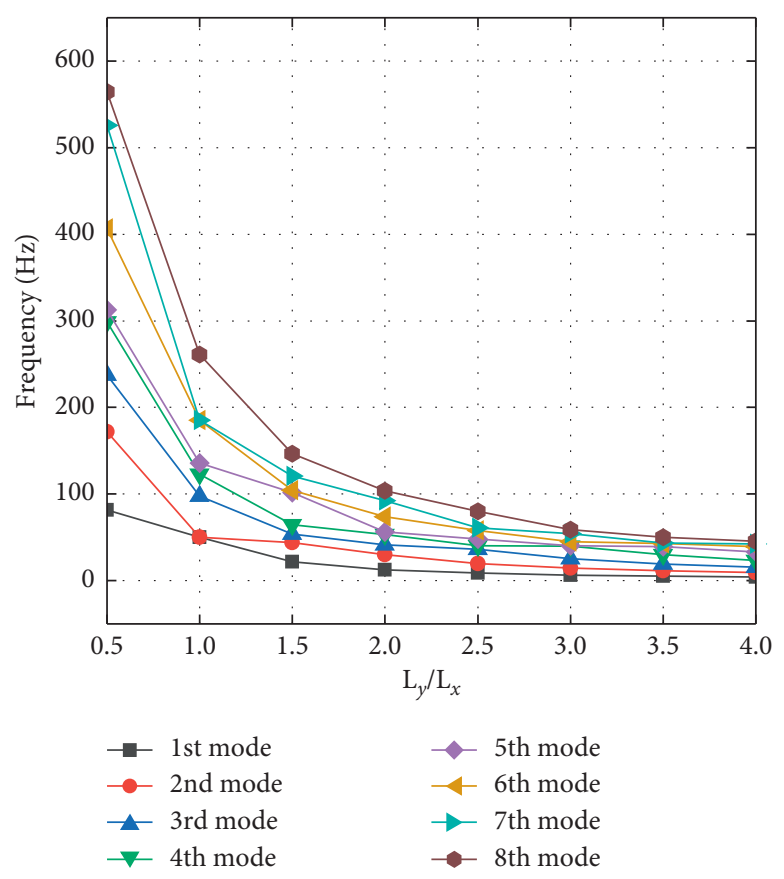

FIGURE 4: Variation of the first eight-order coupling frequency with various length-width ratios.

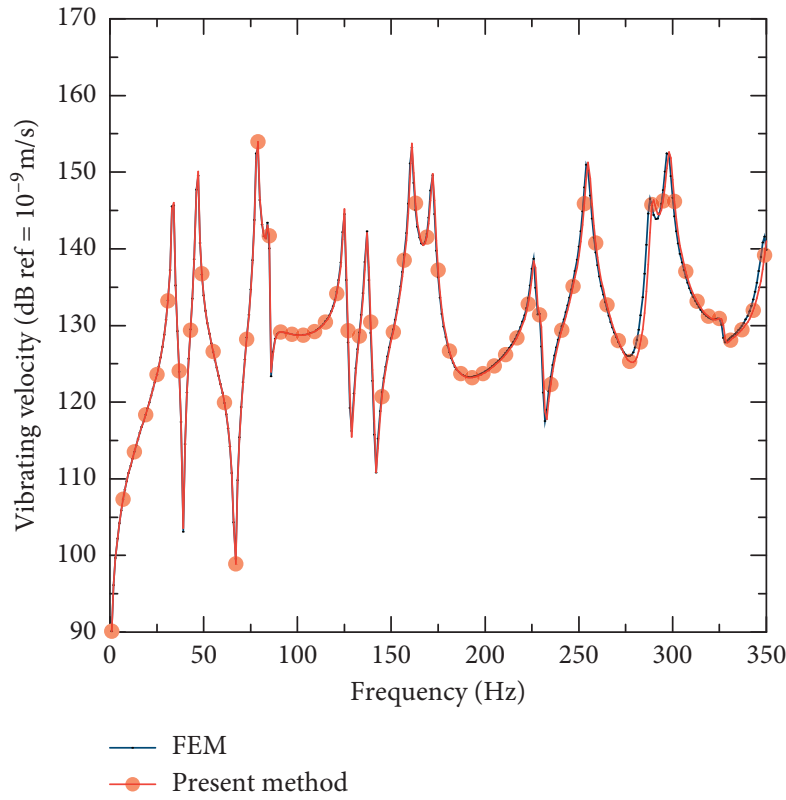

(a)

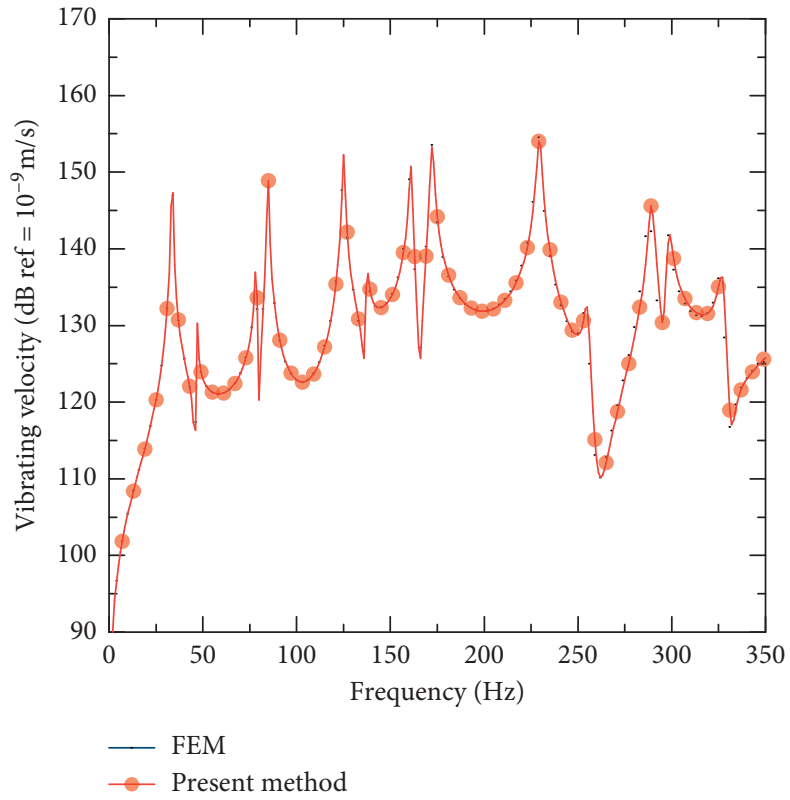

(b)

FIgURE 5: Continued. 


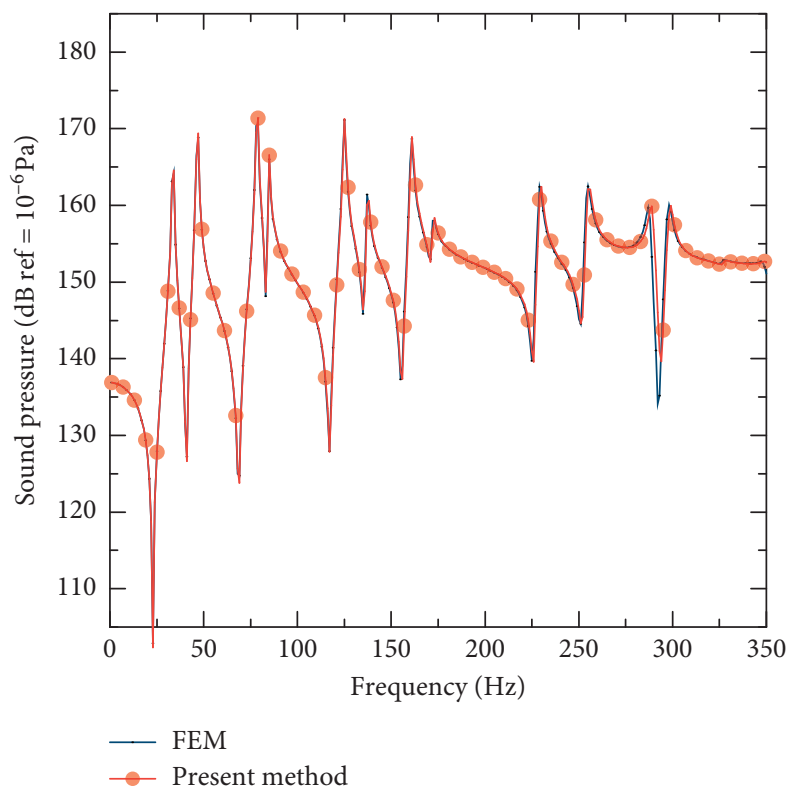

(c)

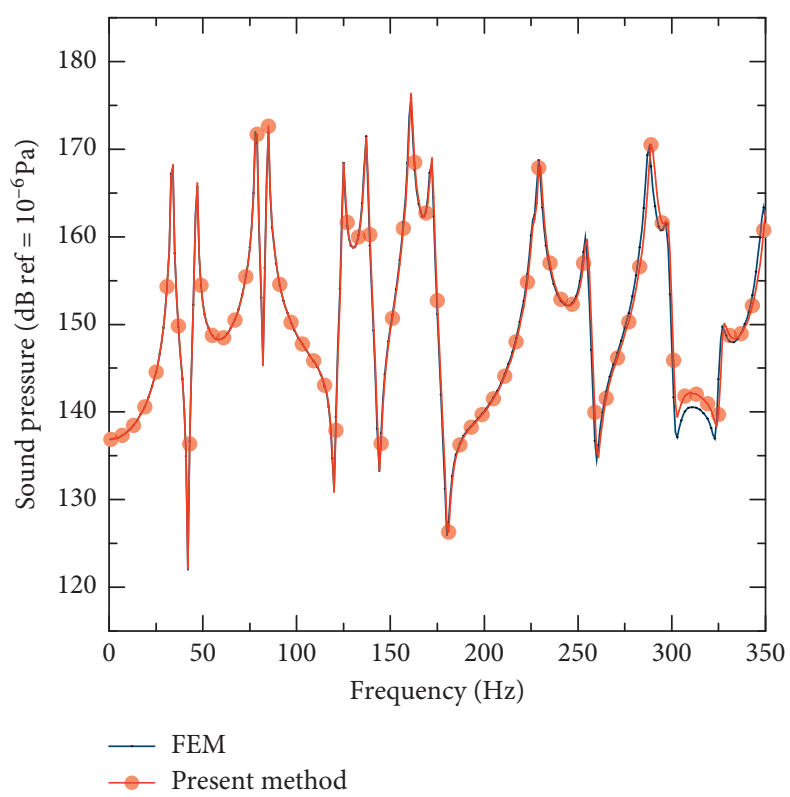

(d)

FigURE 5: Response under point force source excitation. (a) Velocity response at $(0.10,0.12,0.14)$. (b) Velocity response at $(0.15,0.30,0.14)$. (c) Sound pressure response at $(0.08,0.11,0.07)$. (d) Sound pressure response at $(0.20,0.33,0.06)$.

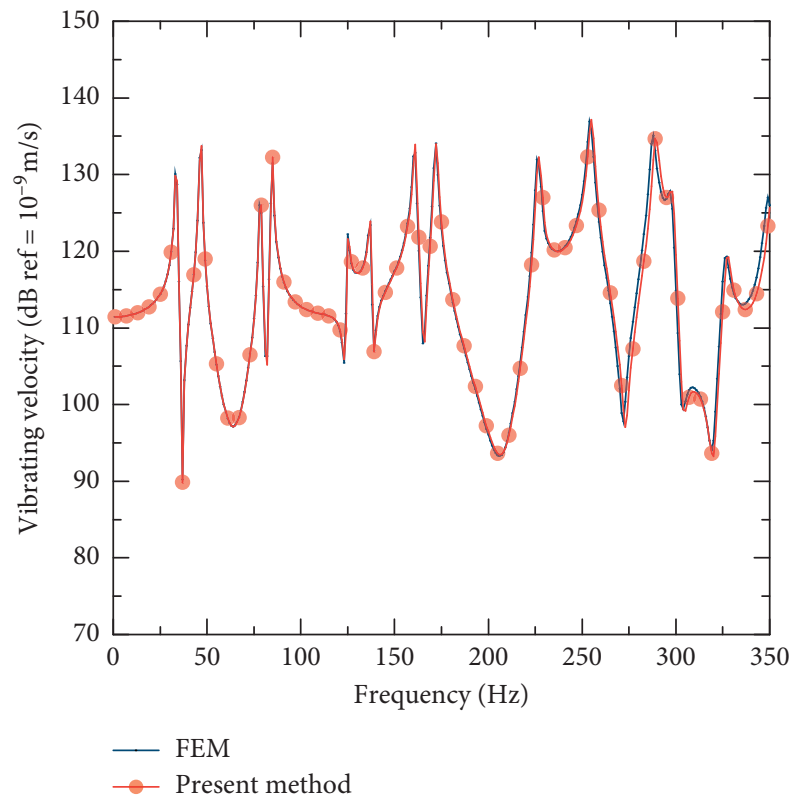

(a)

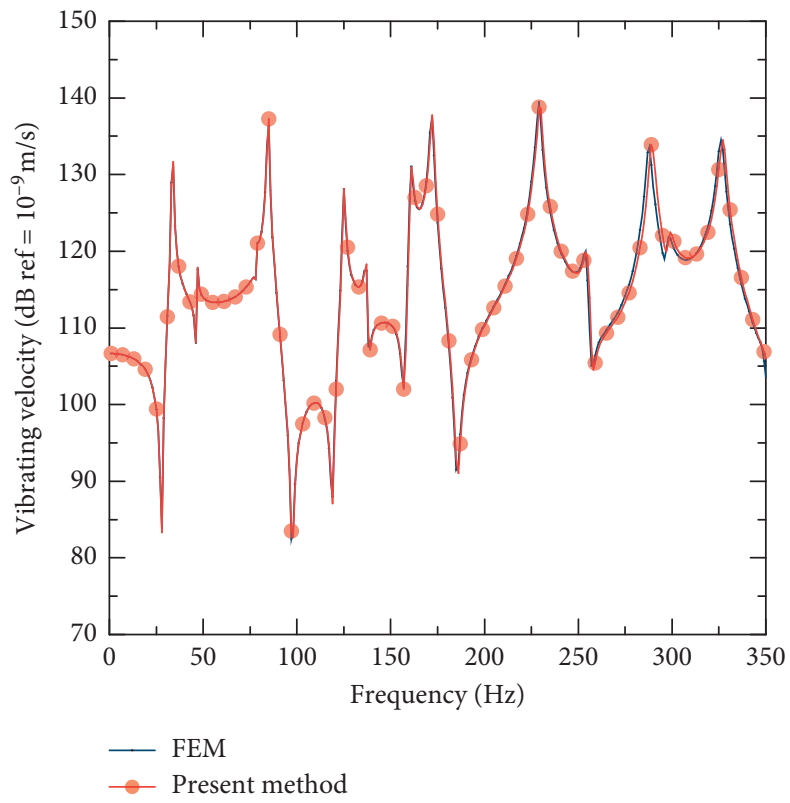

(b)

Figure 6: Continued. 


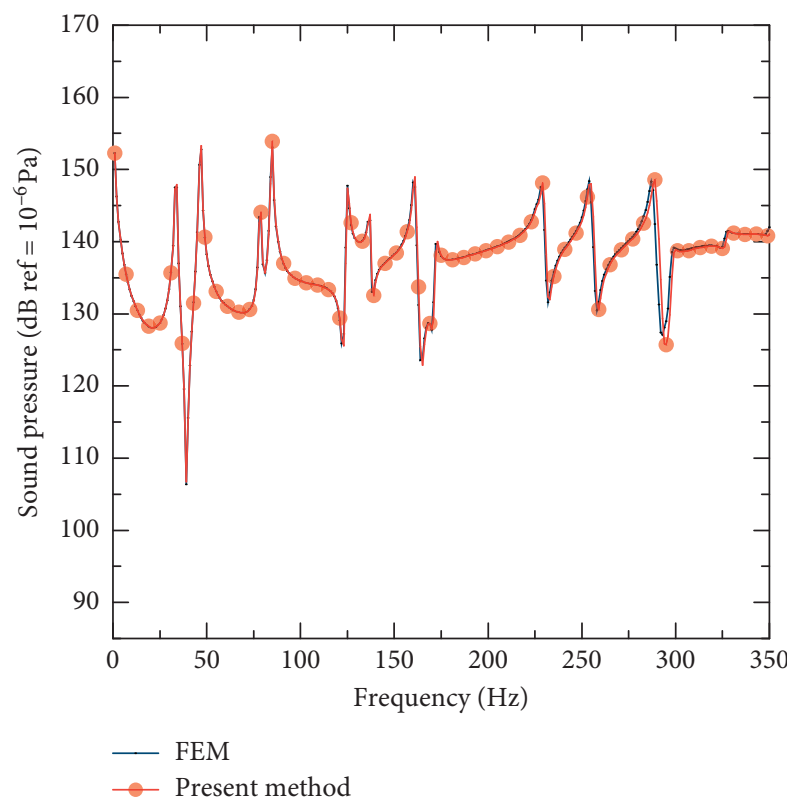

(c)

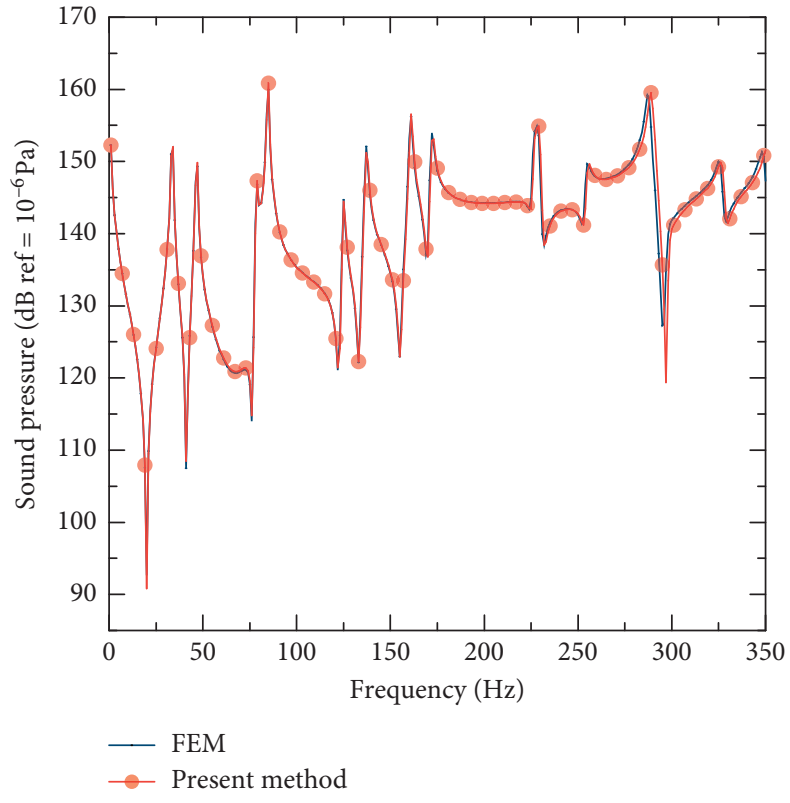

(d)

FIgURE 6: Response under internal point acoustic source excitation. (a) Velocity response at $(0.10,0.12,0.14)$. (b) Velocity response at $(0.15$, $0.30,0.14)$. (c) Sound pressure response at $(0.08,0.11,0.07)$. (d) Sound pressure response at $(0.20,0.33,0.06)$.

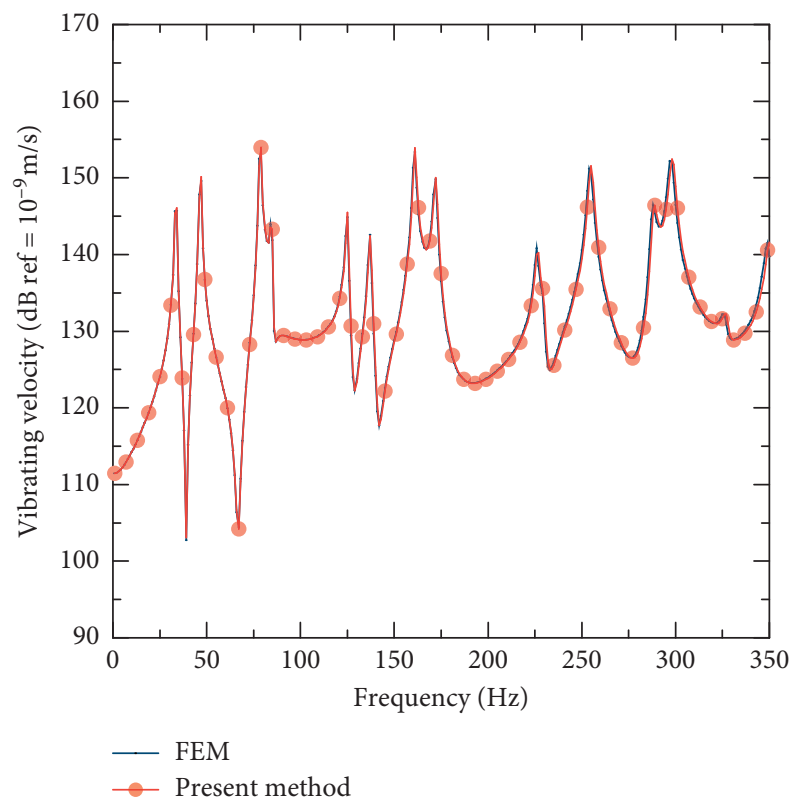

(a)

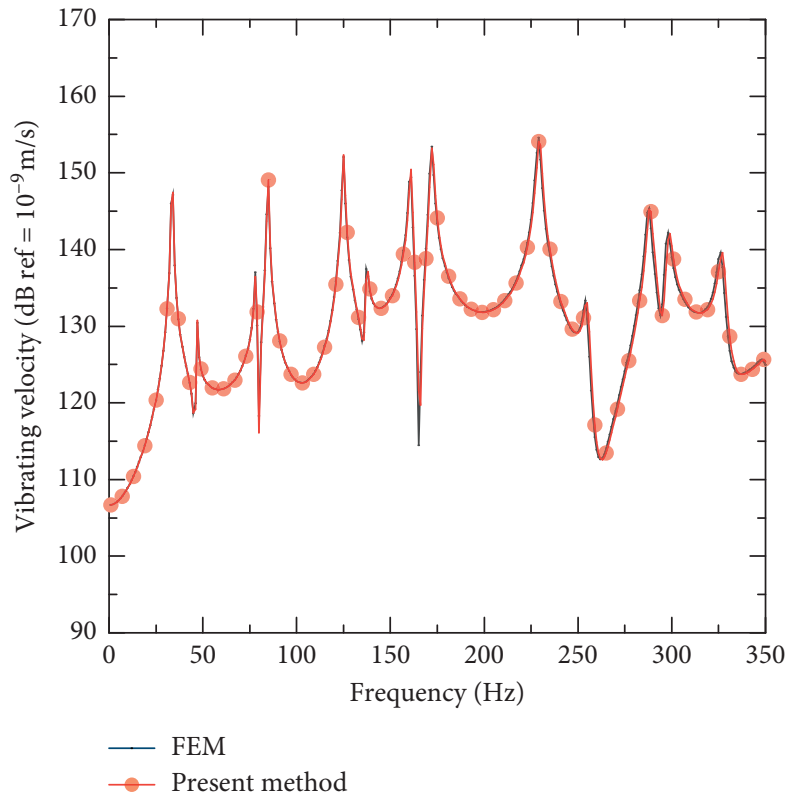

(b)

Figure 7: Continued. 


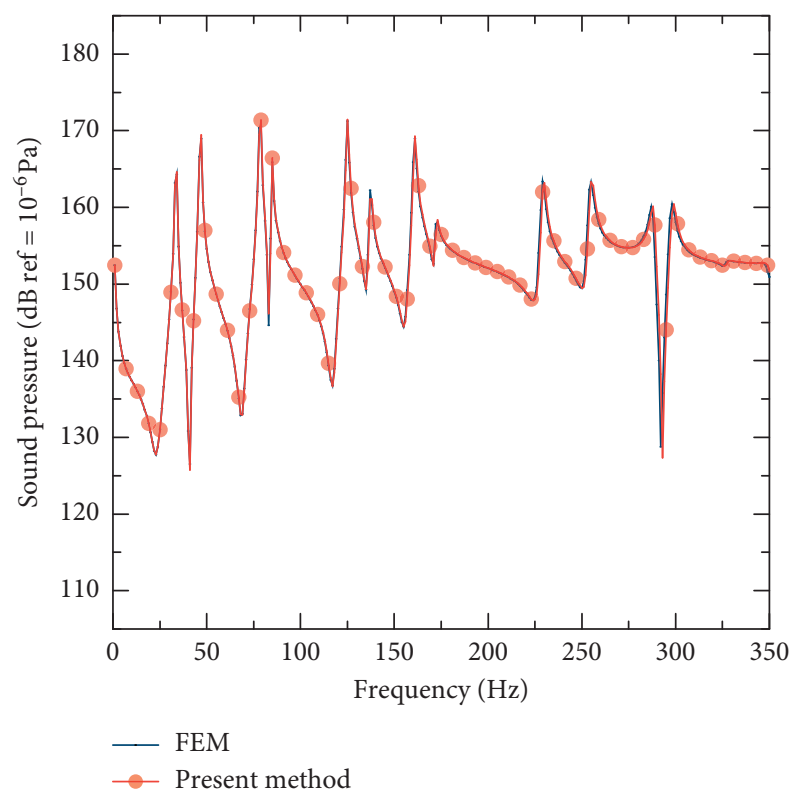

(c)

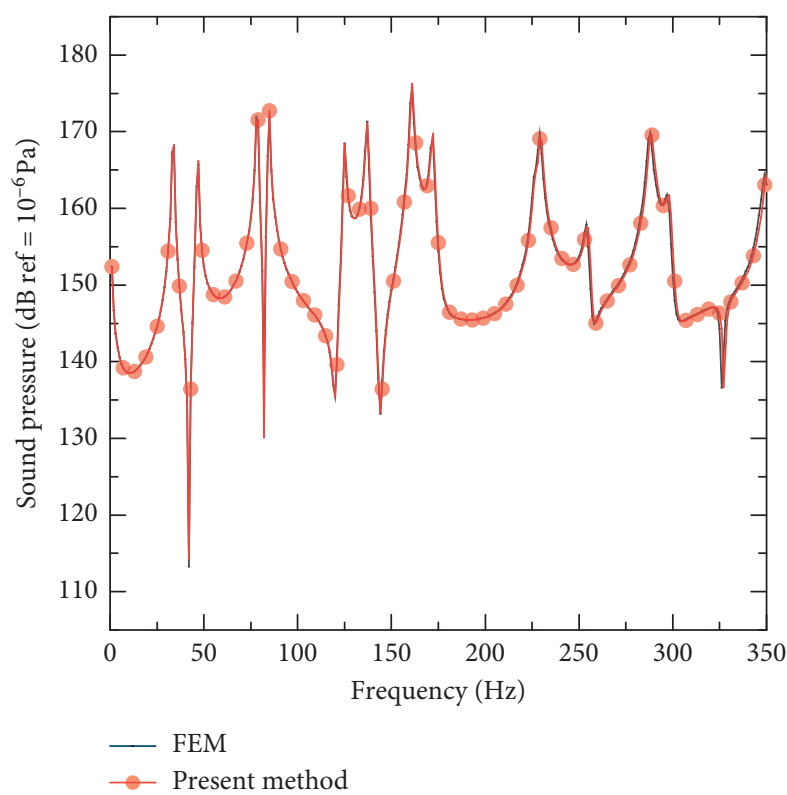

(d)

Figure 7: Response under the combined excitation of point force source and internal point acoustic source. (a) Velocity response at (0.10, $0.12,0.14)$. (b) Velocity response at $(0.15,0.30,0.14)$. (c) Sound pressure response at $(0.08,0.11,0.07)$. (d) Sound pressure response at $(0.20$, $0.33,0.06)$.

excitation, or a combination of both, the response calculation results of the proposed method are in good agreement with the FEM, which also demonstrates the accuracy of the proposed method in predicting the response. Meanwhile, it is feasible to introduce structural damping and acoustic damping by means of complex sound velocity and complex Young's modulus.

\section{Conclusions}

In the paper, a strongly coupled structure-acoustic model for a rectangular cavity and its flexible wall is proposed, and the displacement of the plate and the sound pressure in the cavity are developed based on energy principle theory and Legendre polynomial series. The acoustic characteristics and the forced response of the coupled system are obtained using the Rayleigh-Ritz technique. In addition, the influence of geometric parameters on the acoustic characteristics of the coupled system is analyzed. The results show the following:
(1) The acoustic characteristics of a strongly coupled plate-cavity system can be obtained accurately and quickly, and since the L2 inner product of the Legendre polynomials satisfies orthogonality, the efficiency of the calculation is greatly enhanced.

(2) The change of the structural boundary from clamped supported to simply supported, the reduction of the cavity depth, and the increase in the length-width ratio of the structure all result in a shift of the coupled natural frequencies towards lower frequencies. The property can guide the design of acoustic products. Meanwhile, when the cavity depth is shallow, the coupled natural frequency is sensitive to the change of cavity depth, Therefore, special attention should be paid to cavity depth when designing and applying cavity structures.

(3) The steady-state response under the point force excitation, internal acoustic excitation, and a combination of both can be predicted accurately by the present method, which can provide a new idea for 
active noise control of the strongly coupled plate- Appendix cavity system.

$$
\begin{aligned}
& {\left[K_{m_{\alpha} m_{\beta} m_{\gamma}, m_{\alpha}^{\prime} m_{\beta}^{\prime} m_{\gamma}^{\prime}}^{c}\right]=K_{0}^{c}\left(\Xi_{m_{\alpha} m_{\alpha}^{\prime}}^{1,1} \Psi_{m_{\beta} m_{\beta}^{\prime}}^{0,0} Y_{m_{\gamma} m_{\gamma}^{\prime}}^{0,0}+\Xi_{m_{\alpha} m_{\alpha}^{\prime}}^{0,0} \Psi_{m_{\beta} m_{\beta}^{\prime}}^{1,1} Y_{m_{\gamma} m_{\gamma}^{\prime}}^{0,0}+\Xi_{m_{\alpha} m_{\alpha}^{\prime}}^{0,0} \Psi_{m_{\beta} m_{\beta}^{\prime}}^{0,0} Y_{m_{\gamma} m_{\gamma}^{\prime}}^{1,1}\right),} \\
& {\left[K_{n_{\alpha} n_{\beta}, n_{\alpha}^{\prime} n_{\beta}^{\prime}}^{p}\right]=K_{0}^{p}\left[\Xi_{n_{\alpha} n_{\alpha}^{\prime}}^{2,2} \Psi_{n_{\beta} n_{\beta}^{\prime}}^{0,0}+\Xi_{n_{\alpha} n_{\alpha}^{\prime}}^{0,0} \Psi_{n_{\beta} n_{\beta}^{\prime}}^{2,2}+\eta \Xi_{n_{\alpha} n_{\alpha}^{\prime}}^{2,0} \Psi_{n_{\beta} n_{\beta}^{\prime}}^{0,2}+\eta \Xi_{n_{\alpha} n_{\alpha}^{\prime}}^{0,2} \Psi_{n_{\beta} n_{\beta}^{\prime}}^{2,0}+2(1-\eta) \Xi_{n_{\alpha} n_{\alpha}^{\prime}}^{1,1} \Psi_{n_{\beta} n_{\beta}^{\prime}}^{1,1}\right]} \\
& +\Xi_{n_{\alpha} n_{\alpha}^{\prime}}^{0,0}\left[\bar{k}_{y 0} T_{n_{\beta}}(-1) T_{n_{\beta}^{\prime}}(-1)+\bar{K}_{y 0} T_{n_{\beta}}^{\prime}(-1) T_{n_{\beta}^{\prime}}^{\prime}(-1)\right]+\Xi_{n_{\alpha} n_{\alpha}^{\prime}}^{0,0}\left[\bar{k}_{y L_{y}} T_{n_{\beta}}(1) T_{n_{\beta}^{\prime}}(1)+\bar{K}_{y L_{y}} T_{n_{\beta}}^{\prime}(1) T_{n_{\beta}^{\prime}}^{\prime}(1)\right] \\
& +\Psi_{n_{\beta} n_{\beta}^{\prime}}^{0,0}\left[\bar{k}_{x 0} T_{n_{\alpha}}(-1) T_{n_{\alpha}^{\prime}}(-1)+\bar{K}_{x 0} T_{n_{\alpha}}^{\prime}(-1) T_{n_{\alpha}^{\prime}}^{\prime}(-1)\right]+\Psi_{n_{\beta} n_{\beta}^{\prime}}^{0,0}\left[\bar{k}_{x L_{x}} T_{n_{\alpha}}(1) T_{n_{\alpha}^{\prime}}(1)+\bar{K}_{x L_{x}} T_{n_{\alpha}}^{\prime}(1) T_{n_{\alpha}^{\prime}}^{\prime}(1)\right], \\
& {\left[M_{m_{\alpha} m_{\beta} m_{\gamma}, m_{\alpha}^{\prime} m_{\beta}^{\prime} m_{\gamma}^{\prime}}^{c}\right]=M_{0}^{c} \Xi_{m_{\alpha} m_{\alpha}^{\prime}}^{0,0} \Psi_{m_{\beta} m_{\beta}^{\prime}}^{0,0} \Upsilon_{m_{\gamma} m_{\gamma}^{\prime}}^{0,0}} \\
& {\left[M_{n_{\alpha} n_{\beta}, n_{\alpha}^{\prime} n_{\beta}^{\prime}}^{p}\right]=M_{0}^{p} \Xi_{n_{\alpha} n_{\alpha}^{\prime}}^{0,0} \Psi_{n_{\beta} n_{\beta}^{\prime}}^{0,0}} \\
& {\left[K_{m_{\alpha} m_{\beta} m_{\gamma}, n_{\alpha} n_{\beta}}^{p 2 c}\right]=K_{0}^{p 2 c} \Xi_{n_{\alpha} m_{\alpha}}^{0,0} \Psi_{n_{\beta} m_{\beta}}^{0,0} T_{m_{\gamma}}(1),} \\
& K_{0}^{c}=-\frac{L_{x} L_{y} L_{z}}{2 \rho_{0}}, K_{0}^{p}=4 D L_{x} L_{y}, \bar{k}_{y 0}=\frac{k_{y 0} L_{x}}{2}, \bar{k}_{y L_{y}}=\frac{k_{y L_{y}} L_{x}}{2}, \bar{k}_{x 0}=\frac{k_{x 0} L_{y}}{2}, \\
& \bar{k}_{x L_{x}}=\frac{k_{x L_{x}} L_{y}}{2}, \bar{K}_{y 0}=2 L_{x} K_{y 0}, \bar{K}_{y L_{y}}=2 L_{x} K_{y L_{y}}, \bar{K}_{x 0}=2 L_{y} K_{x 0}, \bar{K}_{x 1}=2 L_{y} K_{x 1} \text {, } \\
& M_{0}^{c}=\frac{L_{x} L_{y} L_{z}}{8 \rho_{0} c_{0}^{2}}, M_{0}^{p}=\frac{\rho_{h} h L_{x} L_{y}}{4}, K_{0}^{p 2 c}=\frac{L_{x} L_{y}}{4}, \quad v, \bar{v}=0,1,2, \\
& \Xi_{a b}^{v, \bar{v}}=\int_{-1}^{1} \frac{d^{v} T_{a}^{v}(\alpha)}{L_{x}^{v} d \alpha^{v}} \frac{d^{\bar{v}} T_{a}^{v}(\alpha)}{L_{x}{ }^{v} d \alpha^{\bar{v}}}\left(a=n_{\alpha}, m_{\alpha} ; b=n_{\alpha}^{\prime}, m_{\alpha}^{\prime}\right), \\
& \Psi_{a b}^{v, \bar{v}}=\int_{-1}^{1} \frac{d^{v} T_{a}^{v}(\beta)}{L_{y}^{v} d \beta^{v}} \frac{d^{\bar{v}} T_{a}{ }^{v}(\beta)}{L_{y}{ }^{v} d \beta^{\bar{v}}}\left(a=n_{\beta}, m_{\beta} ; b=n_{\beta}^{\prime}, m_{\beta}^{\prime}\right), \\
& \Upsilon_{a, b}^{v, \bar{v}}=\int_{-1}^{1} \frac{d^{v} T_{a}^{v}(\gamma)}{L_{z}^{v} d \gamma^{v}} \frac{d^{\bar{v}} T_{a}{ }^{v}(\gamma)}{L_{z}{ }^{v} d \gamma^{\bar{v}}}\left(a=m_{\gamma} ; b=m_{\gamma}^{\prime}\right)
\end{aligned}
$$

where $T_{n_{\alpha}}(\alpha)$ is the $n_{\alpha}^{\text {th }}$ Chebyshev polynomial with the form as $T_{n_{\alpha}}(\alpha)=\cos \left(n_{\alpha} \arccos \alpha\right)$.

\section{Data Availability}

The data that support the findings of this study are available from the corresponding author upon reasonable request.

\section{Conflicts of Interest}

The authors declare that they have no conflicts of interest.

\section{Acknowledgments}

This study was supported by the National Natural Science Foundation of China (no. 51675529).

\section{References}

[1] R. H. Lyon, "Noise reduction of rectangular enclosures with one flexible wall," The Journal of the Acoustical Society of America, vol. 35, no. 11, pp. 1791-1797, 1963.

[2] E. H. Dowell and H. M. Voss, "The effect of a cavity on panel vibration," AIAA Journal, vol. 1, no. 2, pp. 476-477, 1963. 
[3] A. J. Pretlove, "Free vibrations of a rectangular panel backed by a closed rectangular cavity by a closed rectangular cavity," Journal of Sound and Vibration, vol. 2, no. 3, pp. 197-209, 1965.

[4] A. J. Pretlove, "Forced vibrations of a rectangular panel backed by a closed rectangular cavity," Journal of Sound and Vibration, vol. 3, no. 3, pp. 252-261, 1966.

[5] F. J. Fahy, "Vibration of containing structures by sound in the contained fluid," Journal of Sound and Vibration, vol. 10, no. 3, pp. 490-512, 1969.

[6] M. I. Qaisi, "Free vibrations of a rectangular plate-cavity system," Applied Acoustics, vol. 24, no. 1, pp. 49-61, 1988.

[7] J. Pan and D. A. Bies, "The effect of fluid-structural coupling on sound waves in an enclosure-theoretical part," The Journal of the Acoustical Society of America, vol. 87, no. 2, pp. 691-707, 1990.

[8] S. M. Kim and M. J. Brennan, "A compact matrix formulation using the impedance and mobility approach for the analysis of structural-acoustic systems," Journal of Sound and Vibration, vol. 223, no. 1, pp. 97-113, 1999.

[9] H. C. Geng, Z. S. Rao, and Z. S. Han, "New modeling method and mechanism analyses for active control of interior noise in an irregular enclosure using piezoelectric actuators," The Journal of the Acoustical Society of America, vol. 113, no. 3, pp. 1439-1447, 2003.

[10] C. Luo, M. Zhao, and Z. Rao, "The analysis of structuralacoustic coupling of an enclosure using Green's function method," International Journal of Advanced Manufacturing Technology, vol. 27, no. 3-4, pp. 242-247, 2005.

[11] H. Zhang, D. Shi, S. Zha, and Q. Wang, "Vibro-acoustic analysis of the thin laminated rectangular plate-cavity coupling system," Composite Structures, vol. 189, pp. 570-585, 2018.

[12] H. Zhang, R. Zhu, D. Shi, Q. Wang, and H. Yu, "Study on vibro-acoustic property of composite laminated rotary platecavity system based on a simplified plate theory and experimental method," International Journal of Mechanical Sciences, vol. 167, Article ID 105264, 2020.

[13] Y. Y. Lee, "Free vibration analysis of a nonlinear panel coupled with extended cavity using the multi-level residue harmonic balance method," Thin-Walled Structures, vol. 98, pp. 332-336, 2016.

[14] M. Tournour and N. Atalla, "Pseudostatic corrections for the forced vibroacoustic response of a structure-cavity system," The Journal of the Acoustical Society of America, vol. 107, no. 5, pp. 2379-2386, 2000.

[15] J. M. David and M. Menelle, "Validation of a modal method by use of an appropriate static potential for a plate coupled to a water-filled cavity," Journal of Sound and Vibration, vol. 301, no. 3-5, pp. 739-775, 2007.

[16] J. T. Du, W. L. Li, G. Y. Jin, T. Yang, and Z. Liu, "An analytical method for the in-plane vibration analysis of rectangular plates with elastically restrained edges," Journal of Sound and Vibration, vol. 306, no. 3-5, pp. 908-927, 2007.

[17] W. L. Li, X. F. Zhang, J. T. Du et al., "An exact series solution for the transverse vibration of rectangular plates with general elastic boundary supports," Journal of Sound and Vibration, vol. 321, no. 1-2, pp. 254-269, 2009.

[18] J. T. Du, W. L. Li, Z. G. Liu, H. A. Xu, and Z. L. Ji, "Acoustic analysis of a rectangular cavity with general impedance boundary conditions," The Journal of the Acoustical Society of America, vol. 130, no. 2, pp. 807-817, 2011.

[19] J. T. Du, W. L. Li, H. A. Xu, and Z. G. Liu, "Vibro-acoustic analysis of a rectangular cavity bounded by a flexible panel with elastically restrained edges," The Journal of the Acoustical Society of America, vol. 131, no. 4, pp. 2799-2810, 2012.

[20] D. Shi, Y. Zhang, and L. Xiuhai, "Analysis of acoustic characteristics of arbitrary triangular prism and quadrangular prism acoustic cavities," Shock and Vibration, vol. 2019, Article ID 4894610, 17 pages, 2019.

[21] Y. Chen, G. Jin, S. Shi, and Z. Liu, "A general analytical method for vibroacoustic analysis of an arbitrarily restrained rectangular plate backed by a cavity with general wall impedance," Journal of Vibration \& Acoustics, vol. 136, no. 3, Article ID 031015, 2014.

[22] Y. Chen, G. Jin, Z. Feng, and Z. Liu, "Modeling and vibroacoustic analysis of elastically restrained panel backed by irregular sound space," Journal of Sound and Vibration, vol. 409, pp. 201-216, 2017.

[23] S. M. Kim, J.-G. Kim, S.-W. Chae, and K. C. Park, "A strongly coupled model reduction of vibro-acoustic interaction," Computer Methods in Applied Mechanics and Engineering, vol. 347, pp. 495-516, 2019.

[24] C. B. Tucker, Legendre Polynomials and Functions, Create Space, Scotts Valley, CA, USA, 2009.

[25] C. S. Chin and X. Ji, "Analytical modelling of structure-borne sound transmission through I-junction using Chebyshev-Ritz method on cascaded rectangular plate-cavity system," Applied Acoustics, vol. 143, pp. 171-182, 2019.

[26] D. Zhou, Y. K. Cheung, F. T. K. Au, and S. H. Lo, "Threedimensional vibration analysis of thick rectangular plates using Chebyshev polynomial and Ritz method," International Journal of Solids and Structures, vol. 39, no. 26, pp. 6339-6353, 2002.

[27] M. Pirnat, G. Čepon, and M. Boltežar, "Structural-acoustic model of a rectangular plate-cavity system with an attached distributed mass and internal sound source: theory and experiment," Journal of Sound and Vibration, vol. 333, no. 7, pp. 2003-2018, 2014. 